\title{
Effects of Target Temperature Management on the Outcome of Septic Patients with Fever
}

\author{
Ye Gao, ${ }^{1}$ Jianjun Zhu, ${ }^{2}$ Chenyu Yin, ${ }^{1}$ Jianliang $\mathrm{Zhu},{ }^{2}$ Tao Zhu, ${ }^{1}$ and Lijun Liu ${ }^{2}$ \\ ${ }^{1}$ Intensive Care Unit, Taicang Affiliated Hospital of Soochow University, Taicang 215400, China \\ ${ }^{2}$ Intensive Care Unit, The Second Affiliated Hospital of Soochow University, Suzhou 215004, China \\ Correspondence should be addressed to Lijun Liu; lijunliusz@sina.com
}

Received 8 August 2017; Accepted 8 October 2017; Published 12 November 2017

Academic Editor: Hideo Inaba

Copyright (c) 2017 Ye Gao et al. This is an open access article distributed under the Creative Commons Attribution License, which permits unrestricted use, distribution, and reproduction in any medium, provided the original work is properly cited.

\begin{abstract}
Objectives. To investigate the effects of target temperature management on hemodynamic changes, inflammatory and immune factors, and clinical outcomes of sepsis patients with fever. Methods. Patients diagnosed with sepsis with a core temperature of $\geq 39^{\circ} \mathrm{C}$ were randomly divided into two groups: a low-temperature group (LT group: $36.5^{\circ} \mathrm{C}-38^{\circ} \mathrm{C}$ ) and a high-temperature group (HT group: $38.5^{\circ} \mathrm{C}-39.5^{\circ} \mathrm{C}$ ). A target core temperature was achieved within $6 \mathrm{hrs}$ posttreatment and maintained for $24 \mathrm{hrs}$. Then, the hemodynamic changes, inflammatory and immune factors, and clinical outcomes were evaluated. Results. Compared with the HT group, C-reactive protein (CRP), procalcitonin (PCT), interleukin-6 (IL-6), and tumor necrosis factor- $\alpha$ (TNF- $\alpha$ ) showed a significant decrease in the LT group $(P<0.05)$. In contrast, IL-4 and IL-10 were higher in the LT group than in the HT group $(P<0.05)$. The CD4-T lymphocyte $(\mathrm{CD} 4+)$, CD8-T lymphocyte $(\mathrm{CD} 8+)$, and monocytic human leukocyte antigen-DR (mHLADR) in the LT group were higher than in the HT group $(P<0.05)$. The ICU stay and the anti-infection treatment costs were higher in the LT group $(P<0.05)$. Conclusion. Low-temperature management of patients resulted in a low level of proinflammatory cytokines. Excessive temperature control in sepsis patients with fever may be harmful.
\end{abstract}

\section{Introduction}

Sepsis remains a significant healthcare issue that may trigger high mortality and expensive healthcare cost [1]. It may progress into severe sepsis, septic shock, or even multiple organ dysfunction syndrome (MODS) if no treatment options are available immediately after diagnosis [2]. Nowadays, the mortality and prevalence of sepsis are on rise although extensive efforts have been made to better understand its pathogenesis [3].

Hyperthermia $\left(>38^{\circ} \mathrm{C}\right)$ and hypothermia $\left(<36^{\circ} \mathrm{C}\right)$ are the major clinical manifestations for patients with sepsis. In a previous study, Young et al. reported that elevated peak temperature in the first $24 \mathrm{hrs}$ in the ICU was associated with decreased in-hospital mortality in critically ill patients with infection [4]. However, hyperthermia may contribute to tissue and organ damage, which finally results in poor prognosis. For example, Laupland et al. reported that patients with a temperature of $\geq 39.5^{\circ} \mathrm{C}$ showed a higher incidence of arrhythmia, tachycardia, severe brain injury, and even mortality compared with their counterparts with a temperature of $<39.5^{\circ} \mathrm{C}$ [5]. Similarly, hypothermia may also induce deterioration of infection and coagulation disorder and even death as it may inhibit the migration of white blood cells and affect phagocytosis [6]. In septic rats, hypothermia may increase the prevalence of complications after infection compared with the mild hypothermia in septic rats [7]. Additionally, Yang et al. showed that controlling fever to a lower range of $36.0^{\circ} \mathrm{C}-37.5^{\circ} \mathrm{C}$ may induce harmful effects in patients with refractory septic shock with elevation of white blood cells and neutrophils, which implied the decreased capacity of antiinfection when compared to controlling within a higher range of $37.5^{\circ} \mathrm{C}-38.3^{\circ} \mathrm{C}$ [8]. In experimental sepsis rats, postconditioning hypothermia was associated with increased survival duration during experimental sepsis [9]. Moreover, in a clinical multicenter randomized controlled trial, fever control using external cooling was considered to be safe and could 
decrease the vasopressor requirements and early mortality in septic shock fever patients requiring vasopressors [10].

To date, there are still some disputes on temperature control for septic patients with fever. In this study, we aim to identify the temperature range that may benefit septic patients after target temperature management.

\section{Materials and Methods}

2.1. Patients. Septic patients with a core temperature of $\geq 39.0^{\circ} \mathrm{C}$ admitted to the ICU of the Second Affiliated Hospital of Soochow University and Taicang Affiliated Hospital of Soochow University from June 2015 to July 2016 were included in this study. Sepsis was diagnosed according to the Sepsis-3.0 diagnostic standards as previously described [11]. The inclusion criteria were as follows: (i) those diagnosed with sepsis according to the 3.0 standard, (ii) those aged $18-85$ yrs, (iii) those with an expected ICU stay of at least $48 \mathrm{hrs}$, and (iv) those with a core temperature of $\geq 39.0^{\circ} \mathrm{C}$. These patients also comprised those with septic shock. If they met the inclusion criteria, they were included immediately. Otherwise, antishock therapy should be performed according to the guidelines, and then they were included until they met the inclusion criteria. All the exclusion criteria were as follows: (i) those that received defervescence drugs (e.g., nonsteroidal anti-inflammatory drug, NSAID) and/or intravascular cooling; (ii) those with severe cardiac diseases such as obstructive myocardiopathy, myocardial infarction, and ventricular regional wall motion abnormalities; (iii) those that received agents inhibiting the inflammatory mediators (e.g., glucocorticosteroid or ulinastatin); (iv) those with refractory shock [12] as previously described; (v) those that are pregnant; (vi) those with nervous system diseases that may affect the thermoregulatory center, such as cerebral hemorrhage, craniocerebral trauma, intracranial infection, and new cerebral infarction; (vii) those that received renal replacement therapy; and (viii) those who terminated the treatment. All patients signed an informed consent form. The study protocols were approved by the Ethical Committee of the Second Affiliated Hospital of Soochow University and Taicang Affiliated Hospital of Soochow University.

2.2. Grouping. Patients diagnosed with sepsis with a core temperature of $\geq 39^{\circ} \mathrm{C}$ were randomly divided into two groups: a group achieving a "low-temperature" range (LT group: $36.5^{\circ} \mathrm{C}-38^{\circ} \mathrm{C}$ ) and a group achieving a "hightemperature" range (HT group: $38.5^{\circ} \mathrm{C}-39.5^{\circ} \mathrm{C}$ ) by physical methods including a water-flow cooling blanket and ice packs. A target core temperature was achieved within $6 \mathrm{hrs}$ posttreatment and maintained for $24 \mathrm{hrs}$. All the monitoring of core temperature was performed through rectal or blood temperature methods as previously described [13].

2.3. Treatment. The patients were managed according to the guidelines for bundle therapy for sepsis and septic shock as previously described [14]. The major treatment options included fluid resuscitation, vasoactive agents, anti-infective therapy, and other supportive therapies such as analgesics, sedative therapy, respiratory support, blood sugar control, prevention of deep vein thrombosis, nutritional support, and stress ulcer management.

2.4. Data Collection. The data collected included age, sex, admission diagnosis, infection sites, ICU stay, duration and methods of temperature management, Acute Physiology and Chronic Health Evaluation II (APACHE II) score, Sequential Organ Failure Assessment (SOFA) score, drugs for analgesia and sedative therapy, way of ventilation, and temperature monitoring methods.

2.5. Vital Signs and Laboratory Index. The vital signs, circulating monitor indices, routine blood test, bleeding time, coagulation time, infection indices, immune inflammatory factors, blood gas analysis, and blood sugar were determined before temperature management, as well as $12 \mathrm{hrs}$ and $24 \mathrm{hrs}$ after temperature management.

2.6. Hemodynamics and the Use of Vasoactive Agent. The hemodynamics of the patients were monitored using the PICCO system as previously described [15]. The vasoactive agent used for the subjects was noradrenalin, pumped in through a central venous catheter.

2.7. Evaluation of Outcomes. The outcomes evaluated in the study included ICU stay, anti-infection cost, and 28-day survival rate.

2.8. Statistical Analysis. PASW Statistics 18 software was used for the data analysis. Measurement data that are normally distributed were presented as mean \pm standard deviation, while those not normally distributed were presented as median (interquartile). Student's $t$-test or Mann-Whitney $U$ test was used for intergroup comparison. Enumeration data were compared using chi-square test. R-Project software and Bionom.test were used to estimate the $95 \%$ CI of patients' proportion with a $50 \%$ decrease of the vasoactive agent. Prop.test was utilized to compare the difference. Kaplan-Meier method was used to calculate the 28-day survival. Log-rank test was used for the intergroup comparison. $P<0.05$ was considered to be statistically significant.

\section{Results}

3.1. Patient Characteristics. In total, 63 patients (males: 48 , females: 15 , mean age: $58.54 \pm 16.82 \mathrm{yrs}$ ) were included in this study, among whom 31 patients were assigned to the LT group and 32 patients were assigned to the HT group. No statistical differences were noticed in the sex, age, diseases, APACHE II and SOFA scores, infection source, pathogen, and complications between the two groups $(P>0.05$, Table 1$)$. During the $24 \mathrm{hrs}$ of the temperature management, no incidence of severe cardiac arrhythmia or pressure sores was noticed in each group. Eight cases showed shivering, which was mitigated after sedative therapy.

3.2. Changes of Temperature and Hemodynamics at Each Time Point. Before temperature control, no statistical differences were noticed in the core temperature, heart rate, mean arterial 
TABLE 1: Clinical characteristics at baseline.

\begin{tabular}{|c|c|c|c|c|}
\hline Variable & $\begin{array}{l}\text { Low-temperature group } \\
\qquad(n=31)\end{array}$ & $\begin{array}{l}\text { High-temperature group } \\
(n=32)\end{array}$ & $\begin{array}{c}T \text { or } \chi^{2} \text { or Mann-Whitney } \\
U \text { value }\end{array}$ & $P$ value \\
\hline Age (yr) & $56.90 \pm 14.75$ & $60.13 \pm 18.70$ & 0.758 & 0.452 \\
\hline Gender (male/female) & \multirow{2}{*}{$23 / 8$} & \multirow{2}{*}{$25 / 7$} & 0.134 & 0.714 \\
\hline Complication (cases) & & & 1.417 & 0.702 \\
\hline Hypertension & 12 & 9 & & \\
\hline Diabetes & 5 & 7 & & \\
\hline COPD & 4 & 3 & & \\
\hline Old cerebral infarction & 7 & 10 & & \\
\hline APACHE II score & $22(20 \sim 30)$ & $24(21 \sim 31)$ & 399.00 & 0.538 \\
\hline SOFA score & $9(7 \sim 13)$ & $9(6 \sim 13)$ & 491.50 & 0.950 \\
\hline Classification of diseases (cases) & & & 0.176 & 0.674 \\
\hline Medical diseases & 20 & 18 & & \\
\hline Surgical diseases & 11 & 14 & & \\
\hline Infective site & & & 2.816 & 0.412 \\
\hline Lung & 19 & 24 & & \\
\hline Abdominal cavity & 9 & 7 & & \\
\hline Urinary passage & 1 & 1 & & \\
\hline Catheter relevance & 2 & 0 & & \\
\hline Infectious pathogens & & & 0.718 & 0.949 \\
\hline Gram-positive bacteria & 5 & 6 & & \\
\hline Gram-negative bacteria & 15 & 13 & & \\
\hline Fungal infection & 2 & 2 & & \\
\hline Mixed infection & 6 & 6 & & \\
\hline Others & 3 & 5 & & \\
\hline $\begin{array}{l}\text { Number of mechanical ventilation } \\
\text { instances (rate) }\end{array}$ & $11(35.5 \%)$ & $13(40.6 \%)$ & 1.417 & 0.702 \\
\hline Accuracy of anti-infective therapy & & & 1.171 & 0.557 \\
\hline Yes & 24 & 25 & & \\
\hline No & 4 & 2 & & \\
\hline Uncertainty & 3 & 5 & & \\
\hline
\end{tabular}

APACHE II score: Acute Physiology and Chronic Health Evaluation II score; SOFA score: Sequential Organ Failure Assessment score.

pressure (MAP), central venous pressure (CVP), stroke volume (SV), cardiac output (CO), global end-diastolic volume $($ GEDV), and systemic vascular resistance index (SVRI) $(P>$ 0.05 , Figure 1). Within $24 \mathrm{hrs}$ after temperature control, the core temperature, heart rate, $\mathrm{SV}$, and $\mathrm{CO}$ in the LT group were statistically lower than those of the HT group $(P<0.05)$. In contrast, no statistical differences were noticed in the MAP, CVP, GEDV, and SVRI between the LT group and the HT group $(P>0.05)$.

3.3. Changes of Lactic Acid in Each Time Point of Temperature Control. No statistical differences were identified in lactic acid in both groups before temperature control $(P>0.05)$. About $12 \mathrm{hrs}$ and $24 \mathrm{hrs}$ after temperature control, the lactic acid in the LT group was significantly higher than that in the HT group $(P<0.05$, Table 2$)$.

3.4. Comparison of Vasoactive Agent Utilization. At the baseline level, 23 cases received noradrenalin in the LT group and 25 cases received noradrenalin in the HT group, which showed no statistical differences compared with the HT group $(74.2 \%$ versus $78.1 \%, P=0.714)$. Nevertheless, about $24 \mathrm{hrs}$ after temperature control, the proportion of patients with noradrenalin decrease of $50 \%$ compared to the baseline in the HT group was statistically higher than that of the LT group $(P<0.01$, Figure 2$)$.

3.5. Comparison of Inflammatory and Immune Indices. At the baseline level, no statistical differences were noticed in the white blood cell (WBC) count, C-reactive protein (CRP), and procalcitonin (PCT) in both the low-temperature and the high-temperature groups $(P>0.05)$. In contrast, about $24 \mathrm{hrs}$ after temperature control, the WBC, CRP, and PCT in the HT group were statistically higher than in the LT group $(P<0.05$, Table 3).

No statistical differences were identified in IL- 6 and TNF$\alpha$ in both groups before the temperature control $(P>0.05)$, whereas IL- 6 and TNF- $\alpha$ in the LT group showed a significant 


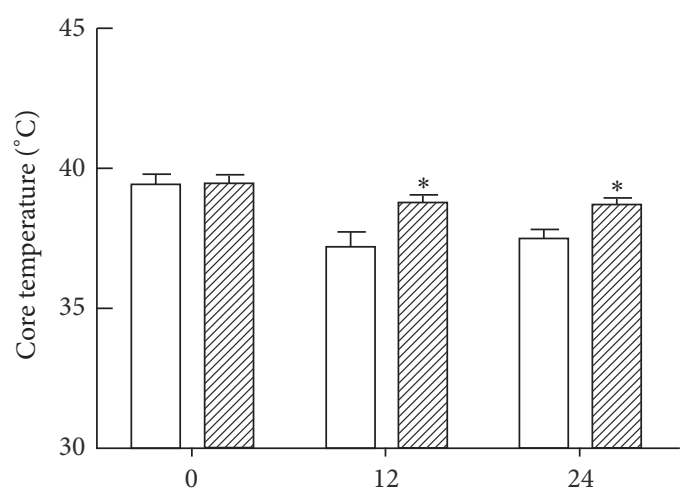

(h)

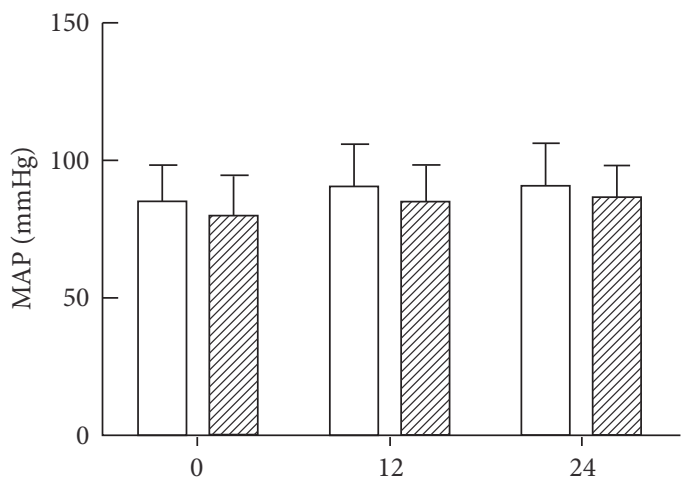

(h)

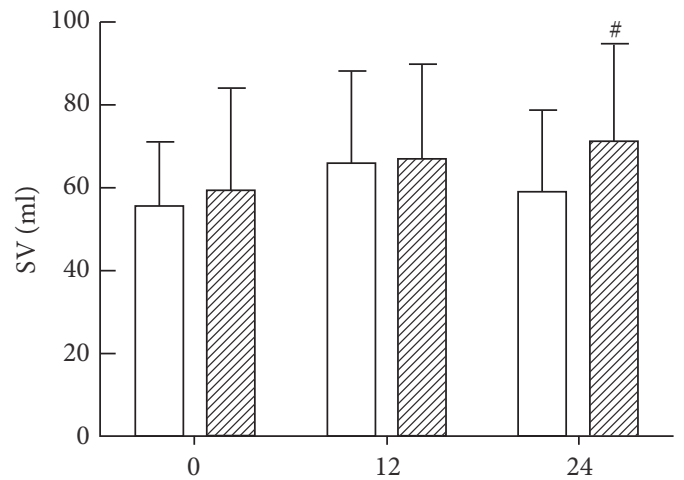

(h)

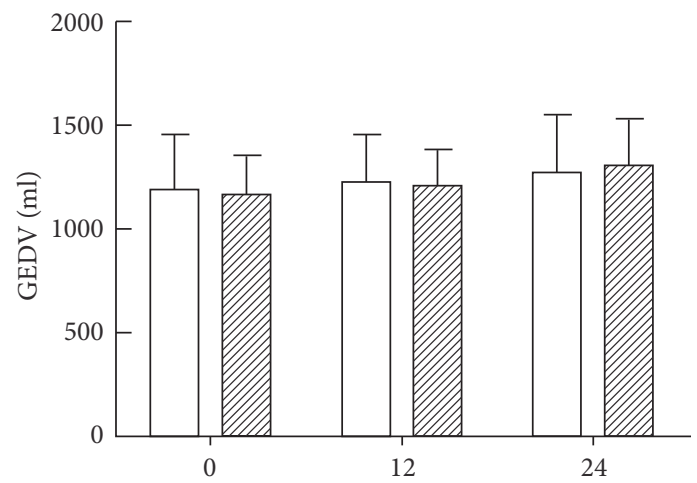

(h)

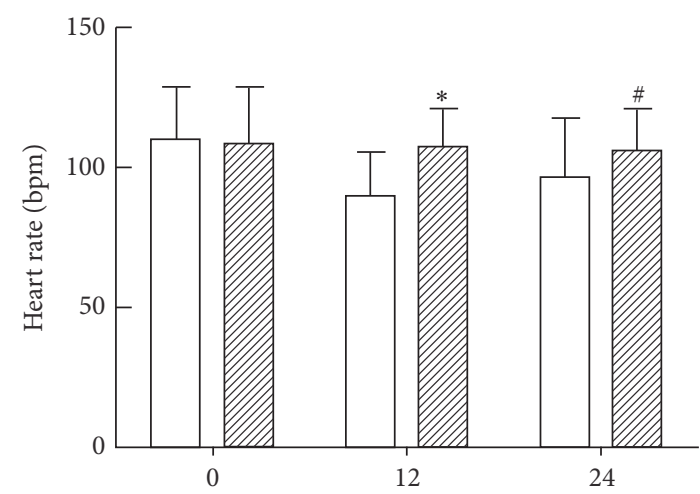

(h)

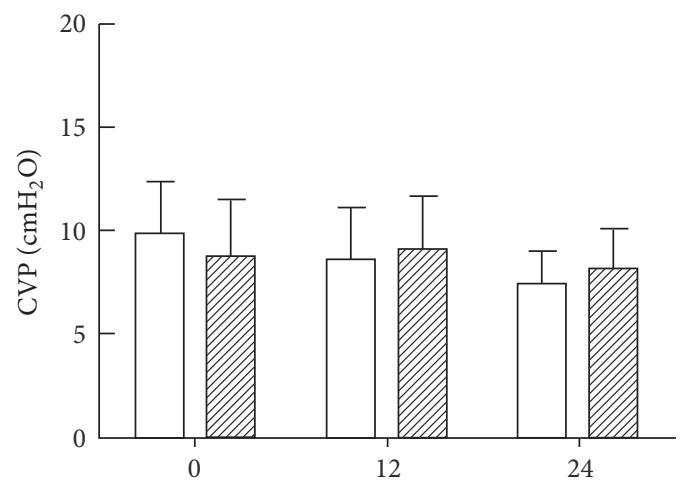

(h)

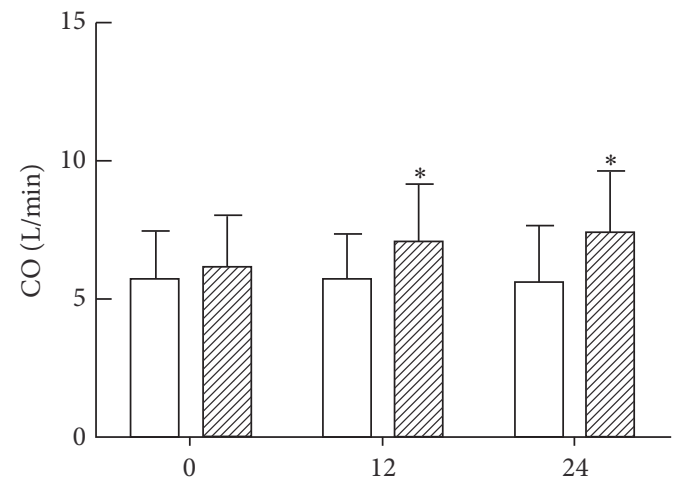

(h)

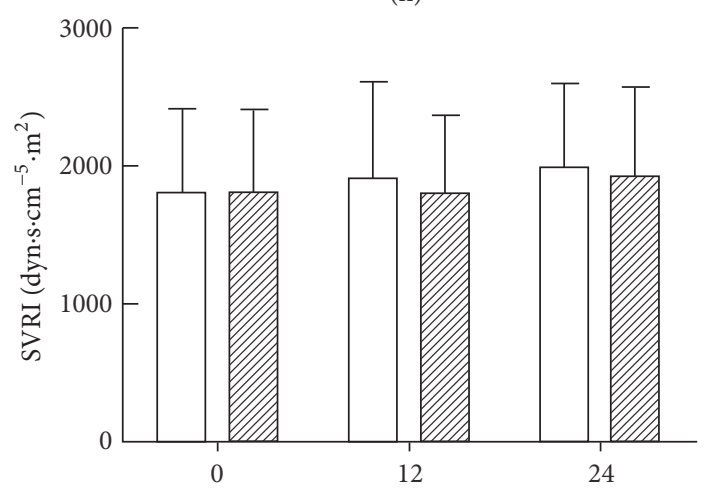

(h)

$$
\begin{array}{llll}
\square & \text { LT group } & \square \text { LT group } \\
\square \text { HT group } & \square \text { HT group }
\end{array}
$$

FIGURE 1: Changes of core temperature, heart rate, MAP, CVP, SV, CO, GEDV, and SVRI in different groups. ${ }^{*} P<0.01$ versus LT group. ${ }^{\#} P<0.05$ versus LT group. LT group: low-temperature group; HT group: high-temperature group. 
TABLE 2: Levels of lactic acid (mmol/L) at different time points in the two groups.

\begin{tabular}{lcccc}
\hline Time $(\mathrm{h})$ & LT group $(n=31)$ & HT group $(n=32)$ & Mann-Whitney $U$ value & $P$ value \\
\hline 0 & $1.20(1.10 \sim 1.60)$ & $1.15(0.83 \sim 2.30)$ & 495.00 & 0.989 \\
12 & $1.75(1.20 \sim 2.43)$ & $1.20(0.70 \sim 1.95)$ & 349.50 & $\mathbf{0 . 0 4 3}$ \\
24 & $1.80(1.18 \sim 2.50)$ & $1.05(0.90 \sim 1.90)$ & 341.50 & $\mathbf{0 . 0 3 3}$ \\
\hline
\end{tabular}

TABLE 3: Changes of WBC, CRP, and PCT at different time points of temperature management.

\begin{tabular}{lccccc}
\hline & Time $(\mathrm{h})$ & LT group $(n=31)$ & HT group $(n=32)$ & Mann-Whitney $U$ value & $P$ value \\
\hline \multirow{2}{*}{ WBC $\left(10^{9} / \mathrm{L}\right)$} & 0 & $15.70(12.30 \sim 26.52)$ & $17.25(10.80 \sim 27.37)$ & 479.00 & 331.00 \\
& 24 & $10.57(6.39 \sim 14.10)$ & $13.90(9.60 \sim 23.08)$ & 467.00 & $\mathbf{0 . 0 2 3}$ \\
\hline \multirow{2}{*}{ CRP $(\mathrm{mg} / \mathrm{L})$} & 0 & $161.51(81.15 \sim 276.51)$ & $164.60(85.68 \sim 205.71)$ & 327.00 & 0.855 \\
& 24 & $89.92(43.08 \sim 144.03)$ & $137.95(76.05 \sim 181.34)$ & 473.50 & 0.031 \\
\hline \multirow{2}{*}{ PCT $(\mu \mathrm{g} / \mathrm{L})$} & 0 & $4.73(2.07 \sim 9.46)$ & $5.42(1.69 \sim 7.51)$ & 341.50 & 0.757 \\
& 24 & $2.65(0.70 \sim 5.65)$ & $5.38(2.36 \sim 12.11)$ & $\mathbf{0 . 0 3 4}$ \\
\hline
\end{tabular}

TABLE 4: Changes of proinflammatory factors at different time points of temperature management.

\begin{tabular}{lccccc}
\hline & Time $(\mathrm{h})$ & LT group $(n=31)$ & HT group $(n=32)$ & Mann-Whitney $U$ value & $P$ value \\
\hline \multirow{2}{*}{ IL-6 $(\mathrm{pg} / \mathrm{ml})$} & 0 & $40.40(13.68 \sim 105.01)$ & $37.19(15.16 \sim 108.76)$ & 491.00 & 0.945 \\
& 12 & $20.19(17.34 \sim 49.04)$ & $43.57(21.33 \sim 114.65)$ & 361.00 & 182.00 \\
\hline & 24 & $17.54(13.11 \sim 98.01)$ & $38.40(29.08 \sim 161.54)$ & $T$ value & 0.045 \\
\hline & Time $(\mathrm{h})$ & LT group $(n=31)$ & HT group $(n=32)$ & 0.450 & 0.010 \\
\hline & 0 & $2.45 \pm 2.33$ & $2.20 \pm 1.96$ & 2.950 & 0.054 \\
TNF- $\alpha(\mathrm{pg} / \mathrm{ml})$ & 12 & $1.56 \pm 0.65$ & $2.11 \pm 0.80$ & $2.46 \pm 1.26$ & 0.037 \\
& 24 & $1.66 \pm 0.96$ & & & 0.006 \\
\hline
\end{tabular}

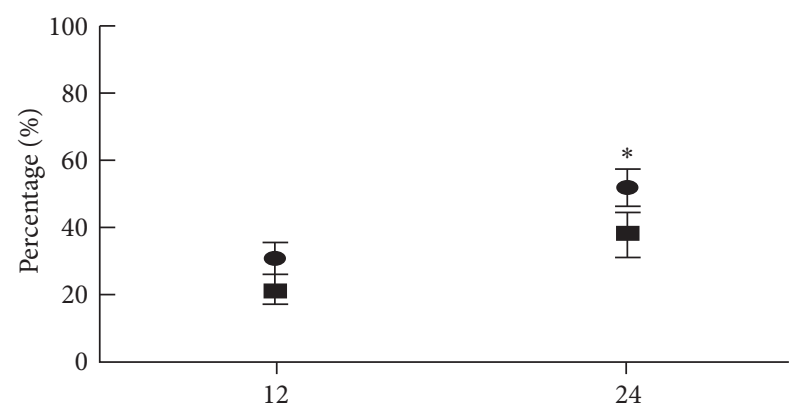

(h)

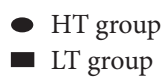

FIGURE 2: Ratio of patients with a decrease of vasoactive agent utilization of $50 \%$ compared to the baseline. ${ }^{*} P<0.01$, comparison between LT group and HT group.

decrease compared with these of the HT group at $12 \mathrm{hrs}$ and $24 \mathrm{hrs}$ after temperature control $(P<0.05$, Table 4$)$.

IL-4 and IL-10 in both groups showed no statistical differences at the baseline levels $(P>0.05)$. Compared with the baseline level, IL-4 in the LT group showed a gradual increase at $12 \mathrm{hrs}$ and $24 \mathrm{hrs}$ after temperature control. Meanwhile, IL-10 reached the peak level at $12 \mathrm{hrs}$, followed by a gradual decrease at $24 \mathrm{hrs}$. In the HT group, both IL- 4 and IL10 showed a trend of decrease. Statistical differences were noticed in IL-4 and IL-10 between the two groups at $12 \mathrm{hrs}$ and $24 \mathrm{hrs}$ after temperature control $(P<0.05$, Table 5$)$.

At the baseline level, the lymphocyte subpopulation (CD4+ and CD8+) and monocytic human leukocyte antigenDR (mHLA-DR) showed no statistical differences in both groups $(P>0.05)$. The lymphocyte subpopulation (CD4+ and $\mathrm{CD} 8+$ ) and mHLA-DR in the LT group showed a gradual increase after temperature control compared with the baseline level $(P<0.05)$. In the HT group, the lymphocyte subpopulation (CD4+ and CD8+) showed no significant difference compared to the baseline level $(P>0.05)$. For the expression of mHLA-DR, a significant increase was noticed in the LT group compared to the control group, while that of the HT group showed a significant decrease compared with the baseline level $(P<0.05$, Figure 3$)$.

3.6. Comparison of ICU Stay and Anti-Infection Therapy Cost. Compared with the HT group, the ICU stay in the LT group showed a significant increase. Similarly, the anti-infection therapy cost in the LT group showed a significant increase compared with that of HT group $(P<0.05$, Figure 4$)$.

3.7. Comparison of 28-Day Survival Analysis. The 28-day survival rate in the LT group was $71.0 \%$, while that in the 
TABLE 5: Changes of anti-inflammatory factors at different time points of temperature management.

\begin{tabular}{lccccc}
\hline & Time $(\mathrm{h})$ & LT group $(n=31)$ & HT group $(n=32)$ & $T$ value & $P$ value \\
\hline \multirow{2}{*}{ IL-4 $(\mathrm{pg} / \mathrm{ml})$} & 0 & $3.64 \pm 0.64$ & $3.63 \pm 0.62$ & $3.56 \pm 0.80$ & 3.753 \\
& 12 & $4.56 \pm 1.27$ & $3.46 \pm 0.65$ & 3.906 & 0.000 \\
& 24 & $4.90 \pm 0.95$ & HT group $(n=32)$ & Mann-Whitney $U$ value & $P$ value \\
\hline & Time $(\mathrm{h})$ & $8.91(6.14 \sim 45.94)$ & $7.75(5.32 \sim 21.75)$ & 449.00 & 0.305 \\
IL-10 $(\mathrm{pg} / \mathrm{ml})$ & 0 & $10.03(5.91 \sim 25.34)$ & $6.96(4.45 \sim 8.91)$ & 325.50 & 0.009 \\
& 12 & $8.80(5.47 \sim 15.82)$ & $5.54(3.98 \sim 6.83)$ & 292.00 & 0.047 \\
\hline
\end{tabular}

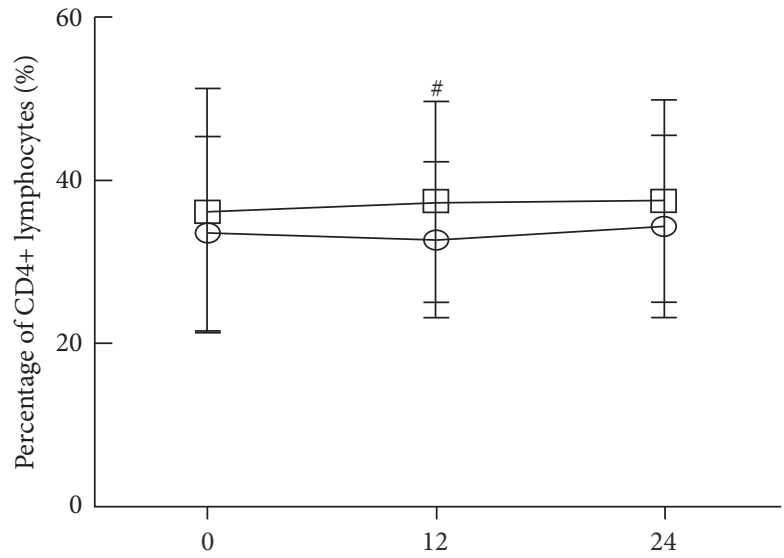

(h)

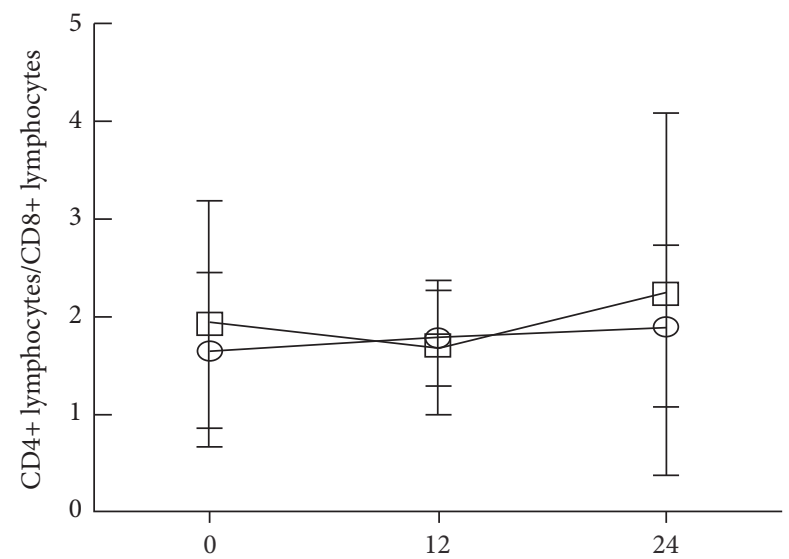

(h)

$\begin{array}{ll}\square & \text { LT group } \\ \ominus & \text { HT group }\end{array}$

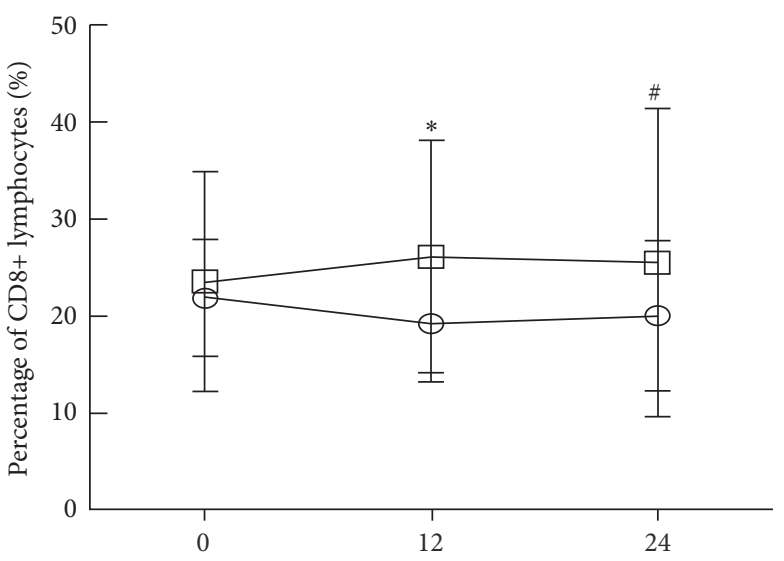

(h)

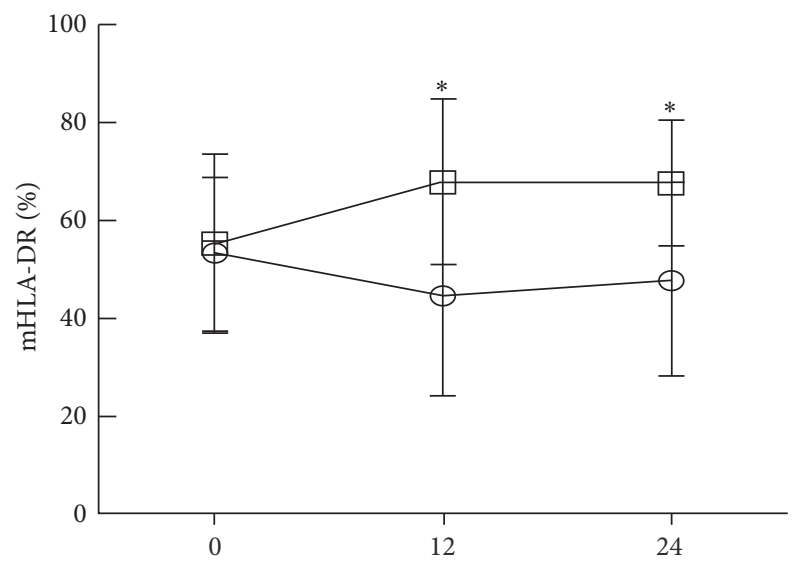

(h)

Figure 3: Changes of lymph node subsets (CD4+ and CD8+) and mHLA-DR antigen in LT group and HT group. ${ }^{*} P<0.01$, comparison between HT group and LT group; ${ }^{*} P<0.05$, comparison between HT group and LT group.

HT group was $81.3 \%$. Using log-rank analysis, no statistical differences were noticed in the 28-day survival rates between the two groups $(P=0.298$, Figure 5$)$.

\section{Discussion}

Fever is an adaptive physiological response to infection and is an important clinical sign in patients with sepsis. In the
ICU, more than $90 \%$ of sepsis patients have fever [16], which is also considered an independent risk factor for death [5, 17]. As an effective strategy for organ protective therapy, body temperature management has been widely used in the treatment of fever [18]. Previous clinical studies reported that body temperature management may affect the survival of patients with sepsis $[19,20]$. For instance, Peres Bota et al. [6] found that patients with septic shock combined 

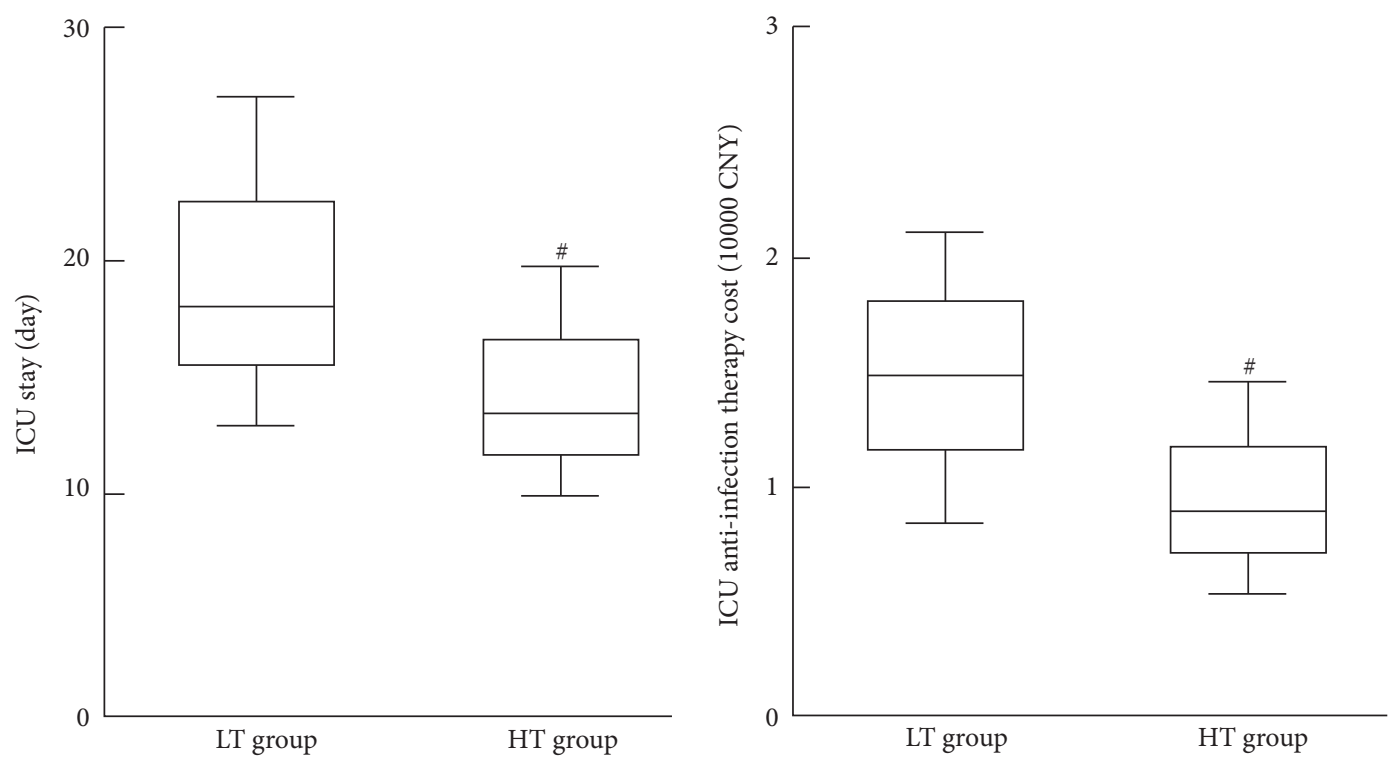

FIgURE 4: ICU hospital stay and cost of anti-infection treatment. ${ }^{*} P<0.05$ versus LT group.

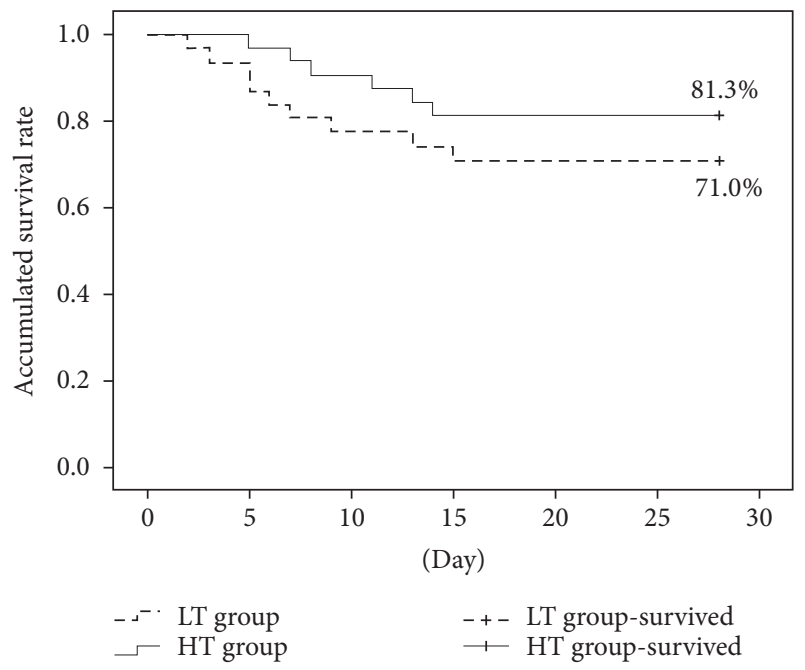

FIGURE 5: The prognosis and 28-day survival rate analysis in the two groups.

with hypothermia showed the highest mortality. Meanwhile, patients with natural hypothermia showed a higher risk of death compared with those with fever [21]. However, there is still a lack of convincing evidence about how patients with sepsis and fever may benefit from temperature management.

An average core temperature of more than $38.3^{\circ} \mathrm{C}$ is defined as fever in sepsis, while a core temperature of more than $39.5^{\circ} \mathrm{C}$ is defined as hyperpyrexia [22]. Fever in sepsis could lead to an increased heart rate and elevation of oxygen demand, while decreased body temperature could reduce the oxygen consumption and energy requirements of the tissues. In our study, patients with hypothermia showed a decreased heart rate and SV, which then led to a decrease of cardiac output directly, thereby reducing tissue perfusion and increasing the risk of poor prognosis. Our study also showed that the levels of blood lactic acid in patients with high temperature were lower than those in patients with lower temperature after temperature management for 24 hours. This indicated that higher $\mathrm{CO}$ and higher oxygen delivery in patients with high temperature caused increased levels of tissue perfusion and promoted the aerobic activity and function recovery of tissues and organs, which finally contributed to the prognosis. Su et al. [23] showed that, in a sheep peritonitis sepsis model, the sheep in the fever group had higher oxygenation index, lower lactic acid level, and longer survival time compared with the sheep in other groups. The normal body temperature of sheep was in the range of $38.0^{\circ} \mathrm{C}-39.0^{\circ} \mathrm{C}$. Therefore, the temperature of $>39.0^{\circ} \mathrm{C}$ was not considered as high fever, but moderate fever. These studies suggested that patients with high temperature may have a better prognosis by means of targeted temperature management.

In addition, in order to investigate whether the dose of vasoactive agent (noradrenaline) could be reduced through target temperature management in sepsis patients with fever, the use of vasoactive agent in the two groups was analyzed in our study. Our results showed that there was no significant difference for the proportion of patients in the use of vasoactive agent in the two groups before temperature management. However, the proportion of patients in whom the dose of vasoactive agent decreased $50 \%$ baseline levels after temperature management for $24 \mathrm{~h}$ was significantly higher in the high-temperature group than in the low-temperature group, which may be related to higher $\mathrm{CO}$ and higher oxygen delivery that cause the easy correction of shock (lower level of lactic acid) in patients with high temperature. However, in a clinical multicenter randomized controlled study, the dose of vasoactive agent was reduced 2 times in septic shock patients with fever after targeted temperature management $\left(36.5^{\circ} \mathrm{C}-37.0^{\circ} \mathrm{C}\right)$ for 48 hours, and the 14 -day mortality was significantly lower [10]. The reasons for this contradictory 
result may be associated with temperature management time and duration and clinical heterogeneity of sepsis patients. Therefore, the relationship between the use of vasoactive drugs and the targeted temperature management in sepsis patients with fever should be further explored.

When sepsis occurs, a network is established between the endogenous inflammatory mediators including vasoactive substances, cytokines, chemokines, oxygen radicals, acute phase reactants, bioactive lipid, plasma enzyme system products, and fibrinolytic pathways. In cases of network imbalance, widespread damage of various systems and organs was induced. Proinflammatory factors such as IL-1, IL6 , and TNF- $\alpha$ could aggravate tissue injury, while antiinflammatory factors such as IL-4, IL-10, and transforming growth factor-beta (TGF- $\beta$ ) could inhibit the inflammatory reaction. The destruction of the dynamic balance between proinflammatory factors and anti-inflammatory factors may be an important mechanism for the development of sepsis. In some experiments, fever could improve the endotoxin effects, and temperature management would affect the changes of inflammation mediators in sepsis. An animal experiment showed that, compared with the normal temperature group $\left(36^{\circ} \mathrm{C}-38^{\circ} \mathrm{C}\right)$, the rats injected with endotoxin had reduced mortality, decreased proinflammatory factors such as IL-6 and TNF- $\alpha$, and decreased NO in the mild-to-moderate lowtemperature $\left(34^{\circ} \mathrm{C}-35^{\circ} \mathrm{C}, 30^{\circ} \mathrm{C}-31^{\circ} \mathrm{C}\right)$ group [24]. Léon et al. [25] found that lowering body temperature could reduce the release of certain inflammatory mediators (IL-6 and TNF- $\alpha$ ) and extent of oxidative response and improve the survival rate in septic rats. Huet et al. [26] revealed that hypothermia $\left(32^{\circ} \mathrm{C}\right)$ could significantly reduce the mortality of rats with endotoxemia, which may be associated with an increased protective effect of the anti-inflammatory factor IL-10. In this study, a decrease of WBC, CRP, PCT, and proinflammatory factors (IL- 6 and TNF- $\alpha$ ) and an increase of anti-inflammatory factors (IL-4 and IL-10) were noticed in the low-temperature group after 24 hours of temperature management, while these indexes had little changes in the high-temperature group after 24 hours of temperature management. This indicated proper reduction of body temperature to $36.0^{\circ} \mathrm{C}-38.5^{\circ} \mathrm{C}$; the endotoxin induced body excessive inflammatory reaction was inhibited, and the survival rate of patients increased. For these reasons, the reduction of body temperature may be related to the decrease of metabolism and energy demands of inflammatory cells, the reduction of proinflammatory cytokines (IL-6 and TNF- $\alpha$ ) release, and the increase of the anti-inflammatory cytokines (IL-4 and IL10) contents.

In addition, our study also observed that there were no significant differences in the levels of lymphocytes subpopulations (CD4+ and CD8+) and mHLA-DR at the beginning of temperature management in the two groups. The levels of lymphocytes subpopulations (CD4+ and CD8+) and mHLADR were increased in the low-temperature group after 24 hours of temperature management. No significant differences were noticed in the levels of CD4+ and CD8+ in the hightemperature group after 24 hours of temperature management, whereas mHLA-DR showed a decreased trend. Lymphocytes are the main cells of the adaptive immune response, which are involved in the innate immune response. They play a vital role in the immune response against pathogenic microorganisms. There is a serious lack of important immune cells such as CD4+ T-cells, CD8+ T-cells, B-cells, dendritic cells, and mononuclear cells in sepsis. HLA-DR is a class MHC-II molecule in the immune index and plays an important role in the monocyte antigen presenting process. The expression level of mHLA-DR is the most commonly used biomarker in the evaluation of the immune status of sepsis, and its persistent low expression is the main feature of immunosuppression stage in sepsis patients [27]. Some studies found that mHLA-DR could be used to assess the prognosis, and its persistent low expression (the positive rate was below $20 \%-40 \%$ ) is also an independent risk factor for septic shock and the incidence of nosocomial infection in ICU hospitalization patients $[28,29]$. However, the lymphocyte subsets (CD4+ and CD8+) and mHLA-DR only reflect part of the body's immune function. The changes of immune status in patients with sepsis are very complex and dynamic, and the heterogeneity among patients is great; therefore, the use of these indicators for the assessment of the overall immune status needs to be discussed.

For the effects of temperature management on the outcome of septic patients, we investigated the ICU stay and anti-infection therapy cost. After achieving a target temperature, the ICU stay in the high-temperature group was comparatively lower than that in the low-temperature group, together with the anti-infection therapy cost for the ICU hospitalization. Meanwhile, the 28-day survival rate in the high-temperature group was higher than that in the lowtemperature group (81.3\% versus $71.0 \%)$. Although no statistical differences were identified $(P=0.298)$, this indicated that septic patients in the high-temperature group showed better outcome and prognosis than those in the lowtemperature group.

There are really some limitations in this study. Septic patients were included in separate ICUs from two different hospitals, which may trigger some differences for the procedures except for temperature management. No subgroup analysis was carried out in this study as the sample size was not large enough, which may affect the outcome to some extent. For the utilization of vasoactive agents, noradrenalin is the major agonist for the $\alpha$ receptor, but it may affect the cardiac $\beta 1$ receptor. However, in this study, we do not analyze the effects of such agent on the heart rate and $\mathrm{CO}$, which may cause differences in the results. For the analysis of 28-day survival rate, no statistical differences were noticed between the two groups which may be related to the small sample size, duration of temperature management, and disease severity.

\section{Conclusions}

Low temperature results in a low level of proinflammatory cytokines in septic patients with fever, and the quantity of lymphocyte subsets becomes high. Meanwhile, the ICU stay in the high-temperature group was comparatively lower than that in the low-temperature group, together with the anti-infection therapy cost for the hospitalization. Excessive temperature control may be harmful to septic patients. 


\section{Conflicts of Interest}

The authors declare that there are no conflicts of interest regarding the publication of this paper.

\section{Authors' Contributions}

Ye Gao and Jianjun Zhu equally contributed to this work.

\section{Acknowledgments}

This study was supported by the Youth Science and Technology Project in Science and Education of Suzhou City (no. KJXW2016059) and a grant from Suzhou Key Medicine Project Fund of China (no. SZXK201504).

\section{References}

[1] M. D. Gonsalves and Y. Sakr, "Early identification of sepsis," Current Infectious Disease Reports, vol. 12, no. 5, pp. 329-335, 2010.

[2] Y. Hattori, K. Hattori, T. Suzuki, and N. Matsuda, "Recent advances in the pathophysiology and molecular basis of sepsisassociated organ dysfunction: novel therapeutic implications and challenges," Pharmacology \& Therapeutics, vol. 177, pp. 5666, 2017.

[3] C. Fleischmann, D. O. Thomas-Rueddel, M. Hartmann et al., "Hospital incidence and mortality rates of sepsis," Deutsches Ärzteblatt International, vol. 113, no. 10, pp. 159-166, 2016.

[4] P. J. Young, M. Saxena, R. Beasley et al., "Early peak temperature and mortality in critically ill patients with or without infection," Intensive Care Medicine, vol. 38, no. 3, pp. 437-444, 2012.

[5] K. B. Laupland, R. Shahpori, A. W. Kirkpatrick, T. Ross, D. B. Gregson, and H. T. Stelfox, "Occurrence and outcome of fever in critically ill adults," Critical Care Medicine, vol. 36, no. 5, pp. 1531-1535, 2008.

[6] D. Peres Bota, F. Lopes Ferreira, C. Mélot, and J. L. Vincent, "Body temperature alterations in the critically ill," Intensive Care Medicine, vol. 30, no. 5, pp. 811-816, 2004.

[7] A. Torossian, S. Ruehlmann, M. Middeke et al., "Deleterious effects of mild hypothermia in septic rats are ameliorated by granulocyte colony-stimulating factor," Anesthesiology, vol. 99, no. 5, pp. 1087-1092, 2003.

[8] Y. L. Yang, D. W. Liu, X. T. Wang, Y. Long, X. Zhou, and W. Z. Chai, "Body temperature control in patients with refractory septic shock: too much may be harmful," Chinese Medical Journal, vol. 126, no. 10, pp. 1809-1813, 2013.

[9] E. L'Her, A. Amerand, A. Vettier, and P. Sebert, "Effects of mild induced hypothermia during experimental sepsis," Critical Care Medicine, vol. 34, no. 10, pp. 2621-2623, 2006.

[10] F. Schortgen, K. Clabault, S. Katsahian et al., "Fever control using external cooling in septic shock: a randomized controlled trial," American Journal of Respiratory and Critical Care Medicine, vol. 185, no. 10, pp. 1088-1095, 2012.

[11] K. Kaukonen, M. Bailey, D. Pilcher, D. J. Cooper, and R. Bellomo, "Systemic inflammatory response syndrome criteria in defining severe sepsis," The New England Journal of Medicine, vol. 372, no. 17, pp. 1629-1638, 2015.
[12] D. Annane, E. Bellissant, and J. Cavaillon, "Septic shock," The Lancet, vol. 365, no. 9453, pp. 63-78, 2005.

[13] J.-Y. Lefrant, L. Muller, J. Emmanuel Coussaye et al., "Temperature measurement in intensive care patients: comparison of urinary bladder, oesophageal, rectal, axillary, and inguinal methods versus pulmonary artery core method," Intensive Care Medicine, vol. 29, no. 3, pp. 414-418, 2003.

[14] R. P. Dellinger, M. M. Levy, A. Rhodes et al., "Surviving sepsis campaign: international guidelines for management of severe sepsis and septic shock: 2012," Critical Care Medicine, vol. 41, no. 2, pp. 580-637, 2013.

[15] R. Cottis, N. Magee, and D. J. Higgins, "Haemodynamic monitoring with pulse-induced contour cardiac output (PiCCO) in critical care," Intensive and Critical Care Nursing, vol. 19, no. 5, pp. 301-307, 2003.

[16] M. M. Arons, A. P. Wheeler, G. R. Bernard et al., "Effects of ibuprofen on the physiology and survival of hypothermic sepsis," Critical Care Medicine, vol. 27, no. 4, pp. 699-707, 1999.

[17] P. S. Barie, L. J. Hydo, and S. R. Eachempati, "Causes and consequences of fever complicating critical surgical illness," Surgical Infections, vol. 5, no. 2, pp. 145-159, 2004.

[18] P. E. Marik, "Fever in the ICU," CHEST, vol. 117, no. 3, pp. 855$869,2000$.

[19] R. Tiruvoipati, K. Ong, H. Gangopadhyay, S. Arora, I. Carney, and J. Botha, "Hypothermia predicts mortality in critically ill elderly patients with sepsis," BMC Geriatrics, vol. 10, article 70, 2010.

[20] K. B. Laupland, H. D. Davies, D. L. Church et al., "Bloodstream infection-associated sepsis and septic shock in critically ill adults: a population-based study," Infection, vol. 32, no. 2, pp. 59-64, 2004.

[21] K. B. Laupland, J.-R. Zahar, C. Adrie et al., "Determinants of temperature abnormalities and influence on outcome of critical illness," Critical Care Medicine, vol. 40, no. 1, pp. 145-151, 2012.

[22] N. P. O’Grady, P. S. Barie, J. G. Bartlett et al., "Guidelines for evaluation of new fever in critically ill adult patients: 2008 update from the American College of Critical Care Medicine and the Infectious Diseases Society of America," Critical Care Medicine, vol. 36, no. 4, pp. 1330-1340, 2008.

[23] F. Su, D. N. Nam, Z. Wang, Y. Cai, P. Rogiers, and J.-L. Vincent, "Fever control in septic shock: beneficial or harmful?" Shock, vol. 23, no. 6, pp. 516-520, 2005.

[24] T. Taniguchi, H. Kanakura, Y. Takemoto, and K. Yamamoto, "Effects of hypothermia on mortality and inflammatory responses to endotoxin-induced shock in rats," Clinical and Diagnostic Laboratory Immunology, vol. 10, no. 5, pp. 940-943, 2003.

[25] K. Léon, C. Moisan, A. Amérand, G. Poupon, and E. L'Her, "Effect of induced mild hypothermia on two pro-inflammatory cytokines and oxidative parameters during experimental acute sepsis," Redox Report, vol. 18, no. 3, pp. 120-126, 2013.

[26] O. Huet, B. Kinirons, L. Dupic et al., "Induced mild hypothermia reduces mortality during acute inflammation in rats," Acta Anaesthesiologica Scandinavica, vol. 51, no. 9, pp. 1211-1216, 2007.

[27] G. Monneret, A. Lepape, N. Voirin et al., "Persisting low monocyte human leukocyte antigen-DR expression predicts mortality in septic shock," Intensive Care Medicine, vol. 32, no. 8, pp. 1175-1183, 2006. 
[28] J. C. Schefold, "Measurement of monocytic HLA-DR (mHLADR) expression in patients with severe sepsis and septic shock: assessment of immune organ failure," Intensive Care Medicine, vol. 36, no. 11, pp. 1810-1812, 2010.

[29] C. Landelle, A. Lepape, N. Voirin et al., "Low monocyte human leukocyte antigen-DR is independently associated with nosocomial infections after septic shock," Intensive Care Medicine, vol. 36, no. 11, pp. 1859-1866, 2010. 


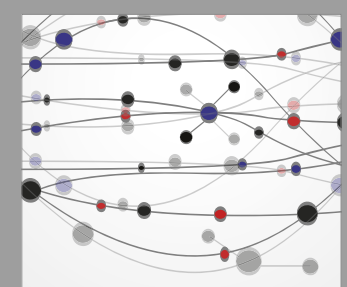

The Scientific World Journal
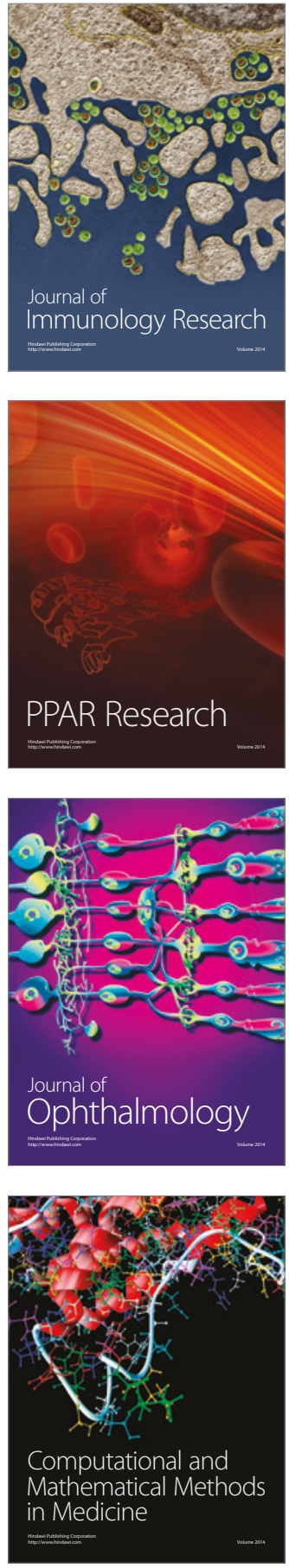

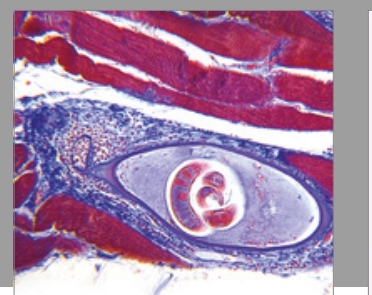

Gastroenterology Research and Practice
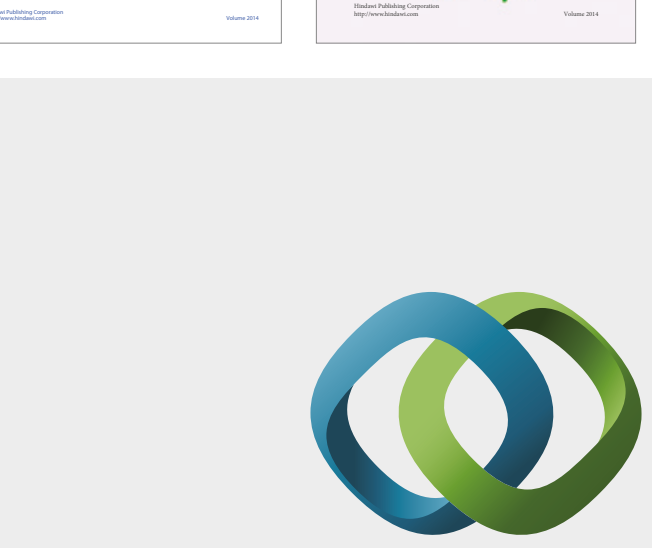

\section{Hindawi}

Submit your manuscripts at

https://www.hindawi.com
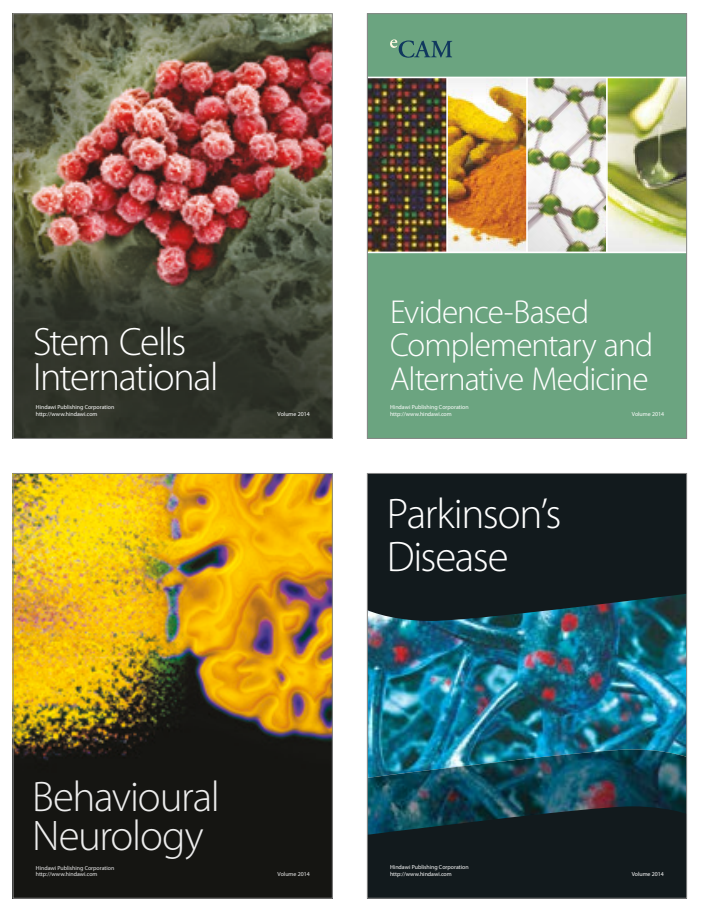
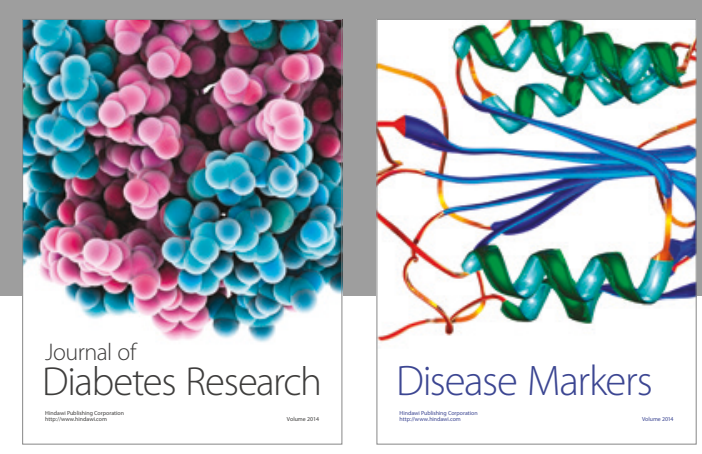

Disease Markers
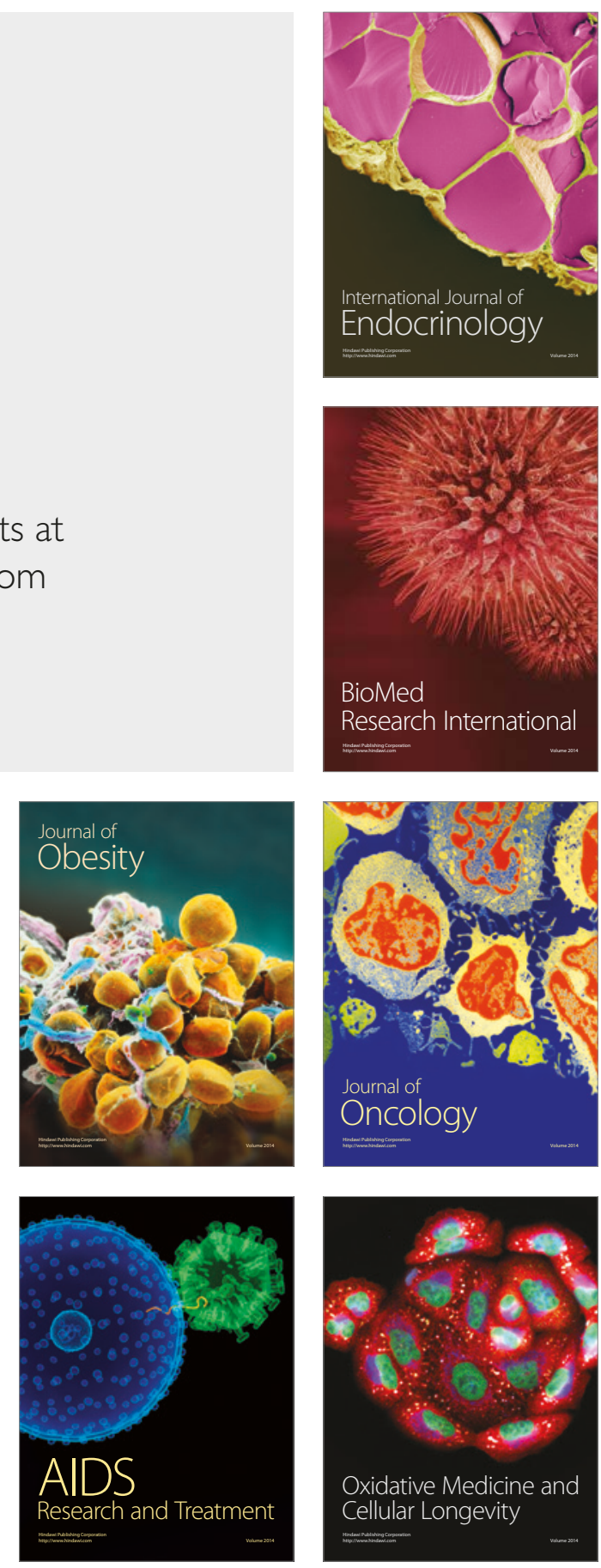